\title{
Routine Screening for Intimate Partner Violence in VHA: A Timely Opportunity
}

\author{
Katherine M. Iverson, Ph.D \\ National Center for PTSD, VA Boston Healthcare System and Boston University School of Medicine, Boston, MA, USA.
}

$\mathrm{J}$ Gen Intern Med 29(2):280

DOI: $10.1007 / \mathrm{s} 11606-013-2716-6$

(c) Society of General Internal Medicine 2013

To the Editors:- Identifying and addressing intimate partner violence (IPV) within the Veterans Health Administration (VHA) is paramount to improving health care and outcomes for our nation's female Veterans. I agree with Drs. Lutwak's $^{1}$ and Feldman's ${ }^{2}$ calls for IPV screening within emergency departments (ED) and primary care (PC) settings, and extend this recommendation to include the need for: (a) integration of IPV screening across VHA medical and mental health settings, (b) emphasis on the importance of accurate and clinically sensitive screening and response, and (c) expansion of VHA infrastructure to provide professional development on best practices in IPV training, screening and assessment, treatment, and referral.

As noted, female Veterans are at high risk for IPV, ${ }^{3}$ which is linked to a multitude of negative health and social consequences. Instituting routine IPV screening in EDs and PCs is imperative, because it would help identify a portion of Veterans in need of IPV services; however, it would miss Veterans not seen in these settings. In order to maximize the benefits of screening and underscore the institutional support for comprehensive IPV care, IPV screening should be integrated more widely into routine care across all VHA medical and mental health settings.

It is also important to ensure that the tools that are used are able to efficiently, accurately, and sensitively identify IPV. Efficient and accurate inquiry is essential, but not sufficient. Empirical and anecdotal evidence indicates a troubling trend of well-intended providers assessing IPV in ways that could discourage disclosure. ${ }^{4}$ IPV screening must be conducted from a trauma-informed approach if we are to see the benefits on health care and health outcomes.
Moreover, it is essential that providers offer appropriate treatment and referrals to address the health and social consequences of IPV. These services may include both VHA and community-based programs, and it is important for providers to be knowledgeable about these resources.

Finally, there is a need for professional development and training regarding IPV screening and response. Both national and local VHA infrastructure could be expanded to enhance guidance available to providers on appropriate procedures for IPV screening, assessment, treatment, and referral. Implementation of IPV education as well as screening and response programs should be accompanied by rigorous evaluations, to allow for modifications and enhancements as needed. VHA has a timely opportunity and is well-positioned to serve as a national model for the implementation of best practices for IPV-related care.

Corresponding Author: Katherine M. Iverson, Ph.D National Center for PTSD, VA Boston Healthcare System and Boston University School of Medicine, 150 South Huntington Avenue (116B-3), Boston, MA, USA (e-mail: Katherine.Iverson@va.gov).

\section{REFERENCES}

1. Lutwak N. Screening for intimate partner violence at VA EDs: the time is now. J Gen Intern Med. 2013; doi:10.1007/s11606-013-2717-5

2. Feldman MD. Screening for intimate partner violence: the time is now. J Gen Intern Med. 2013;28(10):1251-52.

3. Iverson KM, King MW, Resick PA, Gerber MR, Kimerling R, Vogt D. Clinical utility of an intimate partner violence screening tool for female VHA patients. J Gen Intern Med. 2013;28(10):1288-93.

4. Rhodes KV, Frankel RM, Levinthal N, Prenoveau E, Bailey J, Levinson W. "You're not a victim of domestic violence, are you?" Provider patient communication about domestic violence. Ann Intern Med. 2007;147(9):620-27. 